\title{
Application of Linear Model Fitting in Image Edge Fast Detection
}

\author{
Peng Wang Yueming Wang \\ ( school of electronics and information Heilongjiang Institute of science and technology \\ Harbin Heilongjiang 150027,china)
}

\begin{abstract}
A linear model parameter estimation method is proposed based on Bayesian treatment in addition to the linear least squares ,The detail algorithm of one order linear model parameter estimation was introduced in the paper , it can also be used to the parameter estimation of multi-order linear model .we estimate the linear model parameter of image edge use this method .experiment result show that it can finish the parameters fast estimation and the detection of image edge depend on few observed data with a high detect precision.
\end{abstract}

Keywords:linear model; parameter estimation ; edge detection

\section{Introduction}

Linear model fitting is the method that parameter estimation of some model function by observed data ,it has a large application in the image processing and analysis such as high speed currency recognize, and other edge detection area, it is the foundation of image analysis.

Mostly we use the method of linear least squares, many author present some method to the accurency edge detection ,but the algorithm is complex and the amount of calculate and observed data is very large ${ }^{[1][2]}$. we proposed a linear model parameter estimation method based on Bayesian treatment. estimate the linear model parameter of image edge use this method. it can finish the parameters fast estimation and the detection of image edge depend on few observed data with a high detect precision. Use the high speed currency edge detect as the example to discuss the detail of the application of Linear model fitting.

\section{Linear model and parameter estimation}

\subsection{Linear model}

Linear model mean that the model is linear with respect to the model parameters, not( necessarily )with respect to the indicator variables. For example

$$
y_{i}=A_{0}+A_{1} x_{i}+A_{2} x_{i}^{2}+\cdots \text { (1) }
$$

Where $A_{0}, A_{1}, A_{2} \cdots$ are the Linear model parameters of $y_{i}$ and $x_{i}$ is the independent

(indicator)variable .Although $y_{i}$ is not linear with respect to $x_{i}$, there are many similar example in the application ,for example:

$$
\begin{aligned}
& y_{i}=A_{1} \cos \omega t_{i}+A_{2} \sin \omega t_{i} . \\
& \text { we proposed a linear model }
\end{aligned}
$$
parameter estimation method based on Bayesian treatment. it can also be introduce to other area .

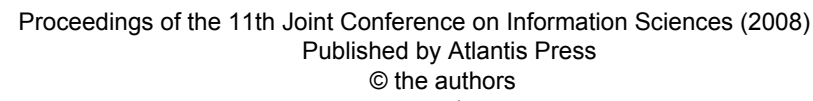




\subsection{Parameter estimation}

We assume that we have $N$ observed data values $d_{i}, i=\{0,1 \cdots, n\}$, that are related to $N$ values of the function $y_{i}$, according to

$$
d_{i}=y_{i}+n_{i}
$$

Where $n_{i}$ represents an unknown error component in the measurement of $y_{i}$,described by Gaussian distribution ,the distribution for each $n_{i}$ to be independent of the value of the other errors and have a common standard deviation $\sigma$.mean value 0 .

By a linear model ,we mean that $y_{i}$ can be written as a linear superposition of $\mathrm{M}$ functions $g_{i \alpha}$,Denoting the coefficients of the known functions by $A_{\alpha}$,we thus have

$$
y_{i}(A)=\sum_{\alpha=1}^{M} A_{\alpha} g_{i \alpha}
$$

Where $\left\{A_{\alpha}\right\}$ is the linear model parameters that we want to estimate, we will sometimes denote collectively with an unadorned $A$,for example

$y_{i}=A_{1}+A_{2} x_{i}+A_{3} x_{i}^{2}+\cdots+A_{M} x_{i}^{M-1}=\sum_{\alpha=1}^{M} A_{\alpha} g_{i \alpha}$

Then $g_{i}=\left\{1, x_{i}, x_{i}^{2}, \cdots, x_{i}^{M-1}\right\}$,called basis functions. According to Bayes's theorem, the purpose of linear model fitting is work out a set of parameters $\left\{A_{\alpha}\right\}$,which can make the joint posterior distribution $p\left(\left\{\mathrm{~A}_{\alpha}\right\} \mid\left\{d_{i}\right\}\right)$ gets the maximum value.

Because $n_{i}$ follow gaussian distribution, $n_{i}=d_{i}-y_{i}$, so the observed data $d_{i}$ follow gaussian distribution too, the joint distribution of $\left\{d_{i}\right\}$ is likelihood function:

$p\left(\left\{\left\{d_{i}\right\} \mid\left\{A_{\alpha}\right\}\right)=\frac{1}{\sigma^{n}(2 \pi)^{\frac{n}{2}}} \exp \left\{\frac{1}{2 \sigma^{2}} \sum_{i=1}^{n}\left(d_{i}-y_{i}\right)^{2}\right]\right.$

Assumed:

$$
\left.Q=\sum_{i=1}^{n}\left(d_{i}-y_{i}\right)^{2}\right]
$$

We insert formula (3) into formula

(6) , Obviously, $Q$ is the function of $\left\{A_{\alpha}\right\}$ :

$$
\begin{aligned}
Q\left(\left\{A_{\alpha}\right\}\right) & \left.=\sum_{i=1}^{n}\left(d_{i}-\sum_{\alpha=1}^{M} A_{\alpha} g_{i \alpha}\right)^{2}\right] \\
& =\sum_{i=1}^{n} d_{i}^{2}+\sum_{i=1}^{n} \sum_{\alpha=1 \beta=1}^{M} A_{\alpha} A_{\beta} g_{i \alpha} g_{i \beta}-2 \sum_{i=1}^{N} d_{i} \sum_{\alpha}^{M} A_{\alpha} g_{i \alpha}
\end{aligned}
$$

Assumed

$$
\begin{aligned}
& \vec{d}=\left[d_{1}, d_{2}, \cdots, d_{N}\right]^{T} \\
& \vec{y}=\left[y_{1}, y_{2}, \cdots, y_{N}\right]^{T} \\
& \vec{g}_{\alpha}=\left[g_{1 \alpha}, g_{2 \alpha}, \cdots, g_{N \alpha}\right]^{T} \\
& \vec{n}=\left[n_{1}, n_{2}, \cdots, n_{N}\right]^{T}
\end{aligned}
$$

formula (2), (3), (7) can be expressed as matrix and vector:

$$
\begin{aligned}
& \vec{d}=\vec{y}+\vec{n} \\
& \vec{y}=\sum_{\alpha=1}^{M} A_{\alpha} \vec{g}_{\alpha}
\end{aligned}
$$

$$
\begin{aligned}
Q\left(\left\{A_{\alpha}\right\}\right) & =(\vec{d}-\vec{y})^{2} \\
& =\vec{d}^{2}+\vec{y}^{2}-2 \vec{d} \cdot \vec{y} \\
& =\vec{d}^{2}+\sum_{\alpha \beta} A_{\alpha} A_{\alpha} \vec{g}_{\alpha} \cdot \vec{g}_{\beta}-2 \sum_{\alpha} A_{\alpha} \vec{d} \cdot \vec{g}_{\alpha} \\
& =\vec{d}^{2}+\sum_{\alpha} A_{\alpha}^{2} \vec{g}_{\alpha}^{2}+2 \sum_{\alpha \neq \beta} A A_{\beta} \vec{g}_{\alpha} \cdot \vec{g}_{\beta}-2 \sum_{\alpha} A_{\alpha} \vec{d} \cdot \vec{g}_{\alpha}
\end{aligned}
$$


In order to make formula (5) get the max, we calculate the Partial Derivative of formula (10) and assumed it equal to 0 :

$\frac{\partial Q\left(\left\{A_{\alpha}\right\}\right)}{\partial A_{\alpha}}=2 \sum_{\beta=1}^{M} A_{\beta} \vec{g}_{\beta} \cdot \vec{g}_{\alpha}-2 \vec{g}_{\alpha} \cdot \vec{d}=0$

formula (11) can be express as linear equations:

$$
\sum_{\beta=1}^{M} A_{\beta} \vec{g}_{\beta} \cdot \vec{g}_{\alpha}=\vec{g}_{\alpha} \cdot \vec{d}
$$

Solve this linear equations, we can obtain the linear model parameters $\left\{A_{1}, A_{2} \cdots, A_{M}\right\}$

\section{Application in line parameter fitting}

We assumed that the line equation need fitting is $y_{i}=A_{1}+A_{2} x_{i}$, observed data vector $\vec{d}$, base function is $\vec{g}_{1}=[1,1, \cdots 1]^{T}$, it include N element, $\vec{g}_{2}=\left[x_{i}, x_{i}, \cdots x_{i}\right]^{T} \quad$ include $\quad \mathrm{N}$ element too, linear equations is:

$$
\left\{\begin{array}{l}
A_{1} \vec{g}_{1} \cdot \vec{g}_{1}+A_{2} \vec{g}_{2} \cdot \vec{g}_{1}=\vec{g}_{1} \cdot \vec{d} \\
A_{1} \vec{g}_{2} \cdot \vec{g}_{1}+A_{2} \vec{g}_{2} \cdot \vec{g}_{2}=\vec{g}_{3} \cdot \vec{d}
\end{array}\right.
$$

where

$$
\begin{gathered}
\vec{g}_{1} \cdot \vec{g}_{1}=\sum_{i=1}^{N} 1=N \\
\vec{g}_{1} \cdot \vec{g}_{2}=\vec{g}_{2} \cdot \vec{g}_{1}=\sum_{i=1}^{N} x_{i} \\
\vec{g}_{2} \cdot \vec{g}_{2}=\sum_{i=1}^{N} x_{i}^{2} \\
\vec{g}_{1} \cdot \vec{d}=\sum_{i=1}^{N} d_{i} \\
\vec{g}_{2} \cdot \vec{d}=\sum_{i=1}^{N} d_{i} \cdot x_{i}
\end{gathered}
$$

insert to formula (13):

$$
\left(\begin{array}{cc}
N & \sum_{i=1}^{N} x_{i} \\
\sum_{i=1}^{N} x_{i} & \sum_{i=1}^{N} x_{i}^{2}
\end{array}\right)\left(\begin{array}{l}
A_{1} \\
A_{2}
\end{array}\right)=\left(\begin{array}{c}
\sum_{i=1}^{N} d_{i} \\
\sum_{i=1}^{N} d_{i} \cdot x_{i}
\end{array}\right)
$$

slove the linear equations:

$$
\begin{array}{r}
A_{1}=\frac{\sum_{i=1}^{N} x_{i}^{2} \sum_{i=1}^{N} d_{i}-\sum_{i=1}^{N} x_{i} \sum_{i=1}^{N} d_{i} \cdot x_{i}}{N \sum_{i=1}^{N} x_{i}^{2}-\left(\sum_{i=1}^{N} x_{i}\right)^{2}} \\
A_{2}=\frac{-\sum_{i=1}^{N} x_{i} \sum_{i=1}^{N} d_{i}+N \sum_{i=1}^{N} d_{i} \cdot x_{i}}{N \sum_{i=1}^{N} x_{i}^{2}-\left(\sum_{i=1}^{N} x_{i}\right)^{2}}
\end{array}
$$

\section{4 experiment result and analysis}

In high speed currency recognition , The currency image is obtained by contact image sensor 。 The currency move speed is $550-600$ sheet $/ \mathrm{min}$, because the high speed movement, image quality is poor and time for processing is very limited, In order to locate the currency image, we must calculate the for currency point accurately 。

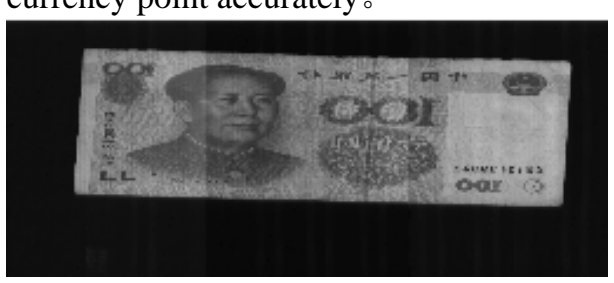

(a)

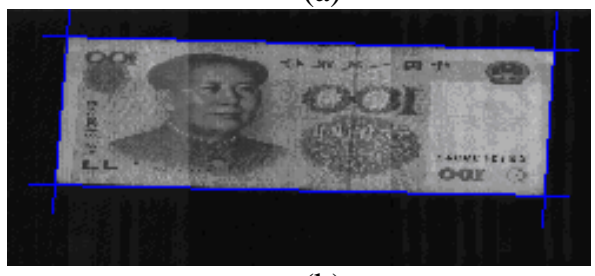

(b)

Figure 1 Currency image edge detect result 
We use formula ( 15 )、 ( 16 ) calculate the four edge line equation , obtain the four currency point coordinate by the four line equation。Through the large amount currency experiment , image resolution is $256 * 110$, we sampling each 10 pixel in horizon , each 5 pixel in vertical to fitting, experiment result show in figure 1,we can obtain each point coordinate by two edge line equation figure (a) is observed image, figure ( $b$ ) is the edge detect result by linear model fitting 。 We label 5300 currency point coordinate manually contrast to the result with this method, as table 1.

\section{5 conclusion}

A linear model parameter estimation method is proposed based on Bayesian treatment. It can also be used to the parameter estimation of multi-order linear model. The method can be used to the image edge detection and other area ..experiment result show that it can finish the parameters fast estimation and the detection of image edge depend on few observed data with a high detect precision.

\section{References}

[1] Han Peiyou , Dong Guiyun , Hao Chongyang, A Bilinear Generalized Fuzzy Enhancement Algorithm to Image Edge Detection Journal of Computer-Aided Design \& Computer Graphics 2005.2

[2] Xing Jian, Liu shengquan , Tian Jun ,Tian Guozhong , A Boundary Points Detector Based On Grid ,Computer Systems \& Applications, 2007.12

Table 1 error analysis of currency edge detect by linear model fiting

\begin{tabular}{c|c|c|c} 
& \multicolumn{2}{c}{ Unit: pixel } \\
& $\left|\Delta x_{\max }\right|$ & $\left|\Delta y_{\max }\right|$ & Point distance max error \\
& 2 & 3 & $\left|D_{\max }\right|$ \\
\hline LUL & 2 & 2 & 3.2 \\
\hline LLL & 3 & 3 & 2.3 \\
\hline RUL & 2 & 2 & 3.6 \\
\hline RLL & Horizontal max error & 2.1 \\
\hline
\end{tabular}

note: because the Horizontalmax error and the vertical max error are not happen in same currency,so point distance max error $\left|D_{\max }\right|$ are no equal to $\sqrt{\Delta x_{\max }^{2}+\Delta y_{\max }^{2}}$. 